Article

\title{
A Novel Synthetic Approach to C-Glycosyl-D- and L-Alanines
}

\section{Miroslava Martinková ${ }^{1, *}$, Jozef Gonda ${ }^{1}$, Jana Raschmanová ${ }^{1}$, Alexandra Novodomská ${ }^{1}$, Jozef Kožíšek ${ }^{2}$ and Lucia Perasinová ${ }^{2}$}

1 Institute of Chemical Sciences, Department of Organic Chemistry, P. J. Šafárik University, Moyzesova 11, SK-040 01 Košice, Slovak Republic; E-mails: jozef.gonda@upjs.sk (J. G.), jrasch@pobox.sk (J. R.), novodomska@pobox.sk (A. N.)

2 Faculty of Chemical and Food Technology, Department of Physical Chemistry, Slovak University of Technology, Radlinského 9, SK-812 37 Bratislava, Slovak Republic; E-mails: jozef.kozisek@stuba.sk (J. K.), lucia.perasinova@stuba.sk (L.P.)

* Author to whom correspondence should be addressed; E-mail: miroslava.martinkova@upjs.sk; Tel.: +421-55 2432329; Fax: +421-55 6222421.

Received: 16 November 2008; in revised form: 10 December 2008 / Accepted: 11 December 2008 / Published: 15 December 2008

\begin{abstract}
C$-Glycosyl- $(S)$ - and $(R)$-alanines 12a and 12b were synthesized from the known $\beta$ - $C$-glycoside 1 . The nitrogen function was introduced by aza-Claisen rearrangement of the allylic thiocyanate 7 , derived from the corresponding alcohol $\mathbf{6}$. The absolute configuration of the newly created chiral carbon center (C-3) was assigned by X-ray diffraction analysis of the intermediate 3(S)-isothiocyanato-D-glycero-D-galacto-decose $8 \mathbf{a}$.
\end{abstract}

Keywords: $\quad C$-Glycosyl amino acids; $C$-Glycosyl alanine; [3,3]-Sigmatropic rearrangements; Microwave irradiation; X-ray diffraction.

\section{Introduction}

Glycoconjugates [1] have a significant pharmaceutical potential and intensive research on understanding the functions of these structures in biological events has become a major target for many scientific groups in the recent years. 
This increasing interest has been recently turned to modified glycosyl amino acids such as $C$ glycosyl $\alpha$-amino acids or fused sugar amino acids [2-5], in which carbohydrate and amino acid are linked directly to the anomeric centre of the sugar either via a carbon-carbon bond or an entire $\alpha$ amino acid (glycinyl moiety) [2]. They represent a significant class of building blocks for the construction of $C$-glycosylated peptides [3,6]. The incorporation of unnatural $C$-glycosyl amino acids in glycopeptide mimetics may serve for preparing analogues with enhanced resistance to enzymatic hydrolysis but also in the development of glycopeptide-based drugs with interesting pharmacological properties [3-5]. For the construction of the $C$-glycosyl amino acids, several synthetic approaches have been developed [2, 5-10].

\section{Results and Discussion}

We report here a synthetic strategy for the preparation of diastereomerically pure $C$-glycosylalanines 12a, 12b, starting from the know $\beta$ - $C$-glycoside 1 [11] and based on the aza-Claisen rearrangement of allylic thiocyanates previously developed in our laboratory [12-13]. The starting $\beta-C$ glycoside 1 was synthesized together with its $\alpha$-anomer 2 via a Wittig-intramolecular Michael-type ring closure sequence from the known 2,3:5,6-di- $O$-isopropylidene- $\alpha$-D-mannofuranose and a stabilized ylide $\left(\mathrm{Ph}_{3} \mathrm{P}=\mathrm{CHCO}_{2} \mathrm{CH}_{3}\right.$, acetonitrile, reflux $)$. Subsequent reduction of $\mathbf{1}$ with lithium aluminum hydride in dry diethyl ether gave alcohol 3 (88\%, Scheme 1). The 2,5-anhydroalditol 3 was subsequently oxidized with $o$-iodoxybenzoic acid [14] (IBX) in acetonitrile to yield the corresponding aldehyde 4 in $93 \%$ yield. The aldehyde 4 was then treated with the stabilized ylide $\mathrm{Ph}_{3} \mathrm{P}=\mathrm{CHCO}_{2} \mathrm{CH}_{3}$ to afford $(E)-\alpha, \beta$-unsaturated ester 5 in $87 \%$ yield (Scheme 1).

Scheme 1. Synthesis of protected 1-thiocyanato-D-glycero-D-galacto-dec-2(E)-enitol 7.

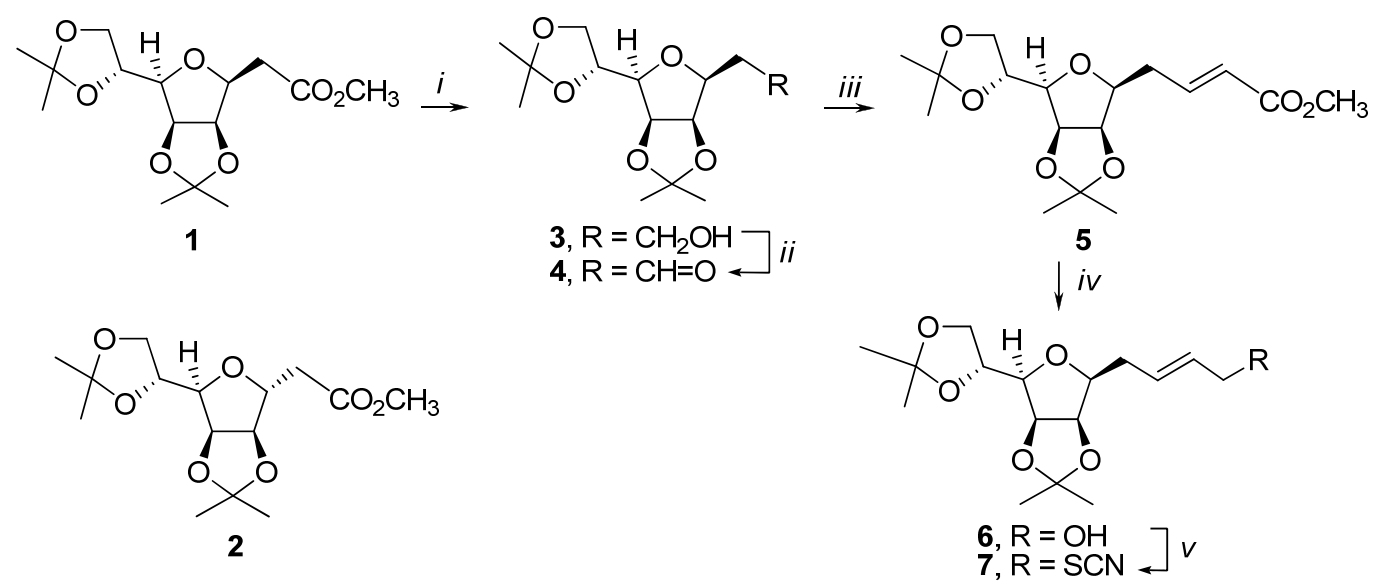

Reagents and conditions: (i) $\mathrm{LiAlH}_{4}, \mathrm{Et}_{2} \mathrm{O}$; (ii) IBX, $\mathrm{CH}_{3} \mathrm{CN}$; (iii) $\mathrm{Ph}_{3} \mathrm{P}=\mathrm{CHCO}_{2} \mathrm{CH}_{3}$, $\mathrm{CH}_{2} \mathrm{Cl}_{2} ;$ (iv) DIBAH, $\mathrm{CH}_{2} \mathrm{Cl}_{2} ;(v) \mathrm{MsCl}, \mathrm{Et}_{3} \mathrm{~N} / \mathrm{KSCN}, \mathrm{CH}_{3} \mathrm{CN}$.

Its structure was determined by ${ }^{1} \mathrm{H}$ - and ${ }^{13} \mathrm{C}-\mathrm{NMR}$ spectroscopy (for data see Experimental part). The observed coupling constant in $\mathbf{5}\left(J_{3,2}=15.7 \mathrm{~Hz}\right)$ accounted for a trans-configuration of the double bond. The ester 5 was subjected to reduction with diisobutylaluminum hydride in $\mathrm{CH}_{2} \mathrm{Cl}_{2}$ to give the allylic alcohol 6 (75\%). The required thiocyanate 7 was easily prepared in $76 \%$ overall yield by a twostep process of mesylation of alcohol $\mathbf{6}$ followed by displacement using KSCN in acetonitrile (Scheme 
1). The thermal aza-Claisen rearrangement of thiocyanate 7 , which was carried out at $90{ }^{\circ} \mathrm{C}$ in dry $n$ heptane under a nitrogen atmosphere for $6 \mathrm{~h}$, afforded a mixture of diastereomeric isothiocyanates 8a and $\mathbf{8 b}$ (Scheme 2$)$, with high yield (83\%) but without selectivity $\left(\mathbf{8 a}: \mathbf{8 b} \approx 1: 1\right.$, as determined by ${ }^{1} \mathrm{H}-$ NMR). The microwave (MW) induced rearrangement of thiocyanate 7 realized under the same conditions $\left(90{ }^{\circ} \mathrm{C}, n\right.$-heptane, Scheme 2$)$ gave a 1:1 mixture of $8 \mathbf{a}$ and $\mathbf{8 b}$ in $86 \%$ yield, within $2 \mathrm{~h}$. The reaction was performed in closed vessel in a focused microwave reactor (CEM Discover, see Experimental part).

Figure 1. A molecular structure of $\mathbf{8 a}$, showing crystallographic numbering.

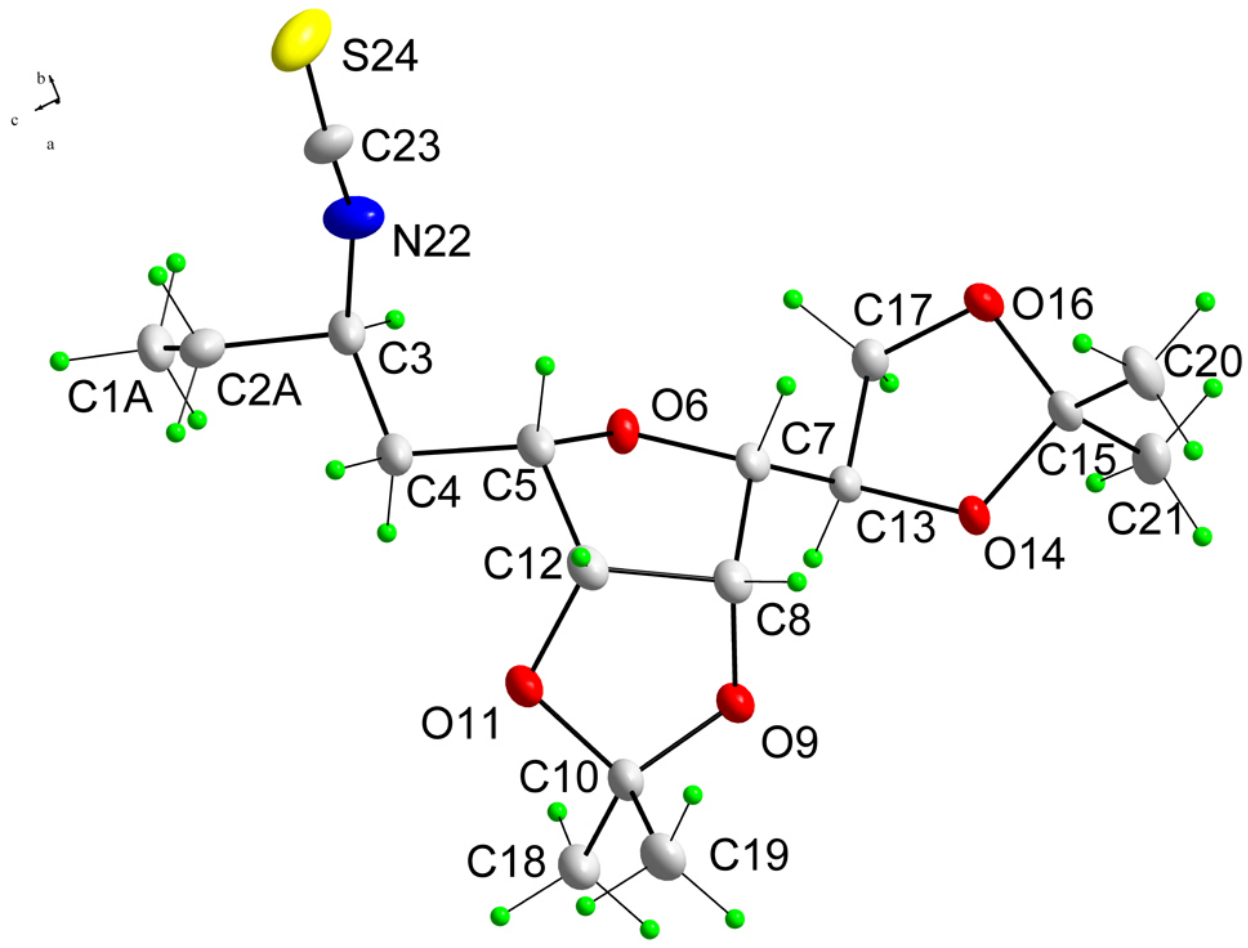

We have observed that the use of microwave irradiation remarkably accelerated rearrangement of $\mathbf{7} \rightarrow \mathbf{8 a}, \mathbf{8 b}$ with reduction to one-third of the reaction time, in comparison with the conventional thermal conditions, but it had practically no influence on the selectivity of the rearrangement.

Fortunately, these diastereoisomers were easily separated by chromatography and compound 8a was isolated in crystalline state. In order to determine the absolute configuration of compound 8a, we tried to recrystallize 8a to obtain single crystals for X-ray diffraction analysis. The isothiocyanate 8a crystallized well from a mixture of ether and hexane, forming colorless prisms suitable for X-ray measurements. The crystallographic structure of compound 8a, shown in Figure 1, confirmed that the newly introduced stereocentre at C-3 in 8a possesses $S$ configuration. Consequently, the isothiocyanate $\mathbf{8 b}$ must be the $3 R$-epimer. 
Scheme 2. Synthesis of $C$-glycosyl- $(S)$ - and $(R)$-alanines.<smiles>C=CC(C[C@@H]1O[C@H]([C@H]2COC(C)(C)O2)[C@H]2OC(C)(C)O[C@H]12)N=C</smiles>

$8 a, 8 b$<smiles>C#CC1(C)OC[C@H]([C@H]2O[C@H](CC(C=C)NC(=O)OC)[C@@H]3OC(C)(C)O[C@H]32)O1</smiles>

9a, $9 b$

$10 \mathrm{a}, 10 \mathrm{~b}$

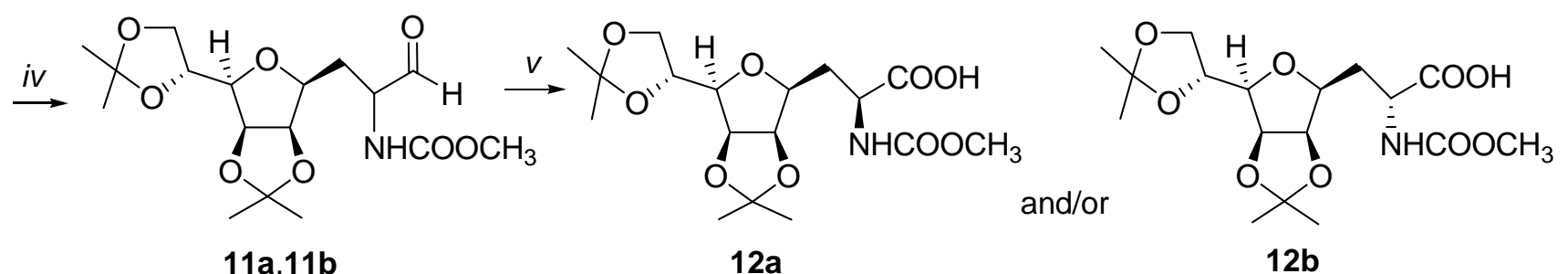

Reagents and conditions: (i) a) $n$-heptane, $90^{\circ} \mathrm{C}$ b) $n$-heptane, $90{ }^{\circ} \mathrm{C}$, microwaves; (ii) $\mathrm{CH}_{3} \mathrm{ONa}, \mathrm{CH}_{3} \mathrm{OH}$; (iii) $\mathrm{MNO}, \mathrm{CH}_{3} \mathrm{CN}$; (iv) $\mathrm{O}_{3},-78{ }^{\circ} \mathrm{C}, \mathrm{Ph}_{3} \mathrm{P}$; (v) $\mathrm{NaClO}_{2}, \mathrm{CH}_{3} \mathrm{CN} / t$ $\mathrm{BuOH} / 2$-methylbut-2-ene $=4: 4: 1$.

Our approach to the build-up of $C$-glycosyl- $(S)$ - and $(R)$-alanines 12a and 12b was based on four subsequent steps which were conducted with pure diastereoisomers $8 \mathbf{a}$ and $\mathbf{8 b}$. In the first step, the reaction of $\mathbf{8 a}$ and $\mathbf{8 b}$ with $\mathrm{CH}_{3} \mathrm{ONa}$ in dry methanol at room temperature gave a nearly quantitative yield of thiourethanes $9 \mathbf{a}$ and $\mathbf{9 b}$, which were used immediately in the next step without purification to avoid problems connected with their possible instability. The treatment of $\mathbf{9 a}$ and $\mathbf{9 b}$ with mesitonitrile oxide (MNO) [15] in acetonitrile afforded in $85 \%$ and $92 \%$ yields, respectively, carbamates $10 a$ and 10b (Scheme 2), whose structure was confirmed by ${ }^{1} \mathrm{H}$ - and ${ }^{13} \mathrm{C}-\mathrm{NMR}$ spectroscopy (for data see experimental part). Ozonolysis of $\mathbf{1 0 a}$ and $\mathbf{1 0 b}$ at $-78{ }^{\circ} \mathrm{C}$ in methanol afforded the corresponding aldehydes 11a and 11b. After a short pad filtration on silica gel (to remove arising triphenylphosphine oxide), these products were used immediately in the next step due to instability of $\alpha$-amino aldehydes. The structure of 11a and 11b was determined by ${ }^{1} \mathrm{H}-\mathrm{NMR}$; the observed chemical shift of aldehyde proton in 11a $\delta=9.64 \mathrm{ppm}$ and in 11b $\delta=9.52 \mathrm{ppm}$. The aldehydes 11a and 11b were selectively oxidized to protected $C$-glycosyl- $(S)$ - and $(R)$-alanines 12a and 12b (Scheme 2) by treatment with sodium chlorite $\left(\mathrm{NaClO}_{2}\right)$ in $\mathrm{CH}_{3} \mathrm{CN} /$ tert-butyl alcohol/2-methyl-2-butene at $0{ }^{\circ} \mathrm{C}$ in $74 \%$ and $73 \%$ yields, respectively after flash chromatography.

\section{Conclusions}

In summary, the novel synthetic approach to the chiral non-racemic $C$-glycosylated alanines 12a and $\mathbf{1 2 b}$ has been developed. The obtained compounds 12a and 12b differ in the stereochemistry of the newly formed chiral carbon atom $(\mathrm{C}-2)$, one having the L-configuration (12a) and the other the Dconfiguration (12b). These novel amino acids $\mathbf{1 2} \mathbf{a}$ and $\mathbf{1 2 b}$ can be useful in modifying the properties of 
some glycopeptides by virtue of the presence of a stable anomeric C-C bond instead of the C-O or C-N bond and an additional amino group at C-2.

\section{Experimental}

\section{General}

All commercially available reagents were used without purification and solvents were dried according to standard procedures. Product purification was carried out using flash chromatography on silica gel (Merck silica gel $60(0.040-0.063 \mathrm{~mm})$. TLC was run on Merck silica gel $60 \mathrm{~F}_{254}$ analytical plates; detection was carried out with either UV, iodine and spraying with a solution of $\mathrm{KMnO}_{4}$, with subsequent heating. The melting points were determined on the Kofler block, and are uncorrected. Optical rotations were measured in chloroform, using a P3002 Krüss polarimeter and reported as follows: $[\alpha]_{\mathrm{D}}{ }^{25}(c$ in $\mathrm{g} / 100 \mathrm{~mL}$, solvent). NMR spectra were recorded at room temperature on a FT NMR spectrometer Varian Mercury Plus $400\left({ }^{1} \mathrm{H}\right.$ at $400.13 \mathrm{MHz}$ and ${ }^{13} \mathrm{C}$ at $\left.100.6 \mathrm{MHz}\right)$ using $\mathrm{CDCl}_{3}$ as the solvent and TMS as internal reference. For ${ }^{1} \mathrm{H} \delta$ are given in parts per million relative to TMS ( 0 ppm), for ${ }^{13} \mathrm{C}$ relative to $\mathrm{CDCl}_{3}(77 \mathrm{ppm}) .{ }^{13} \mathrm{C}-\mathrm{NMR}$ multiplicities were determined by a DEPT pulse sequence. IR spectra were recorded on a Perkin-Elmer 599 IR spectrometer in $\mathrm{CHCl}_{3}$. All reactions were performed under nitrogen atmosphere when anhydrous solvents were used. Microwave experiments were conducted using a focused microwave system (CEM Discover). All experiments were performed in glass vessels $(10 \mathrm{~mL})$ sealed with a septum. At the end of reaction, the vessels and contents were cooled rapidly using a stream of compressed air.

Methyl 3,6-anhydro-2-deoxy-4,5:7,8-di-O-isopropylidene-D-glycero-D-galacto-octanoate (1) and Methyl 3,6-anhydro-2-deoxy-4,5:7,8-di-O-isopropylidene-D-glycero-D-talo-octanoate (2). $\quad \mathbf{1}:{ }^{1} \mathrm{H}$ NMR: $\delta 1.33\left(3 \mathrm{H}, \mathrm{s}, \mathrm{CH}_{3}\right), 1.37\left(3 \mathrm{H}, \mathrm{s}, \mathrm{CH}_{3}\right), 1.44\left(3 \mathrm{H}, \mathrm{s}, \mathrm{CH}_{3}\right), 1.46\left(3 \mathrm{H}, \mathrm{s}, \mathrm{CH}_{3}\right), 2.73(1 \mathrm{H}$, dd, $\left.J_{2,2}=16.7 \mathrm{~Hz}, J_{3,2}=6.4 \mathrm{~Hz}, \mathrm{H}_{2}\right), 2.81\left(1 \mathrm{H}, \mathrm{dd}, J_{2,2}=16.7 \mathrm{~Hz}, J_{3,2}=7.3 \mathrm{~Hz}, \mathrm{H}_{2}\right), 3.52\left(1 \mathrm{H}, \mathrm{m}, \mathrm{H}_{6}\right), 3.70$ $\left(3 \mathrm{H}, \mathrm{s}, \mathrm{OCH}_{3}\right), 3.94\left(1 \mathrm{H}, \mathrm{m}, \mathrm{H}_{3}\right), 4.04\left(1 \mathrm{H}, \mathrm{dd}, J_{8,8}=8.7 \mathrm{~Hz}, J_{8,7}=4.7 \mathrm{~Hz}, \mathrm{H}_{8}\right), 4.07\left(1 \mathrm{H}, \mathrm{dd}, J_{8,8}=8.7 \mathrm{~Hz}\right.$, $\left.J_{8,7}=6.1 \mathrm{~Hz}, \mathrm{H}_{8}\right), 4.38\left(1 \mathrm{H}, \mathrm{ddd}, J_{7,6}=7.5 \mathrm{~Hz}, J_{8,7}=6.1 \mathrm{~Hz}, J_{8,7}=4.7 \mathrm{~Hz}, \mathrm{H}_{7}\right), 4.76\left(2 \mathrm{H}, \mathrm{m}, \mathrm{H}_{4}, \mathrm{H}_{5}\right) ;{ }^{13} \mathrm{C}-$ NMR: $\delta 24.6,25.2,25.7,26.9,33.3,51.8,66.9,73.1,77.7,80.7,81.0,81.6,109.1,112.6,171.4$. The procedure and $[\alpha]_{\mathrm{D}}$ were consistent with those reported [11]. 2: ${ }^{1} \mathrm{H}-\mathrm{NMR}: \delta 1.34\left(3 \mathrm{H}, \mathrm{s}, \mathrm{CH}_{3}\right), 1.37$ $\left(3 \mathrm{H}, \mathrm{s}, \mathrm{CH}_{3}\right), 1.45\left(3 \mathrm{H}, \mathrm{s}, \mathrm{CH}_{3}\right), 1.51\left(3 \mathrm{H}, \mathrm{s}, \mathrm{CH}_{3}\right), 2.47\left(1 \mathrm{H}, \mathrm{dd}, J_{2,2}=15.2 \mathrm{~Hz}, J_{3,2}=7.1 \mathrm{~Hz}, \mathrm{H}_{2}\right), 2.54$ $\left(1 \mathrm{H}, \mathrm{dd}, J_{2,2}=15.2 \mathrm{~Hz}, J_{3,2}=7.7 \mathrm{~Hz}, \mathrm{H}_{2}\right), 3.71\left(3 \mathrm{H}, \mathrm{s}, \mathrm{OCH}_{3}\right), 3.79\left(1 \mathrm{H}, \mathrm{dd}, J_{7,6}=7.7 \mathrm{~Hz}, J_{6,5}=3.7 \mathrm{~Hz}\right.$, $\left.\mathrm{H}_{6}\right), 4.00\left(1 \mathrm{H}, \mathrm{dd}, J_{8,8}=8.7 \mathrm{~Hz}, J_{8,7}=4.4 \mathrm{~Hz}, \mathrm{H}_{8}\right), 4.08\left(1 \mathrm{H}, \mathrm{dd}, J_{8,8}=8.7 \mathrm{~Hz}, J_{8,7}=6.3 \mathrm{~Hz}, \mathrm{H}_{8}\right), 4.39(1 \mathrm{H}$, ddd, $\left.J_{7,6}=7.7 \mathrm{~Hz}, J_{8,7}=6.3 \mathrm{~Hz}, J_{8,7}=4.4, \mathrm{H}_{7}\right), 4.49\left(1 \mathrm{H}, \mathrm{dd}, J_{3,2}=7.7 \mathrm{~Hz}, J_{3,2}=7.1 \mathrm{~Hz}, \mathrm{H}_{3}\right), 4.64(1 \mathrm{H}, \mathrm{d}$, $\left.J_{5,4}=6.0 \mathrm{~Hz}, \mathrm{H}_{4}\right), 4.81\left(1 \mathrm{H}, \mathrm{dd}, J_{5,4}=6.0 \mathrm{~Hz}, J_{6,5}=3.7 \mathrm{~Hz}, \mathrm{H}_{5}\right) ;{ }^{13} \mathrm{C}-\mathrm{NMR}: \delta 24.7,25.2,26.1,27.0,36.2$, $51.9,66.9,73.3,80.8,80.9,80.9,84.9,109.2,112.9,170.6$. The procedure, m.p. and $[\alpha]_{\mathrm{D}}$ were consistent with those reported [11].

3,6-Anhydro-2-deoxy-4,5:7,8-di-O-isopropylidene-D-glycero-D-galacto-octitol (3): $\mathrm{LiAlH}_{4}(0.87 \mathrm{~g}$, $23.0 \mathrm{mmol})$ was added at $0{ }^{\circ} \mathrm{C}$ to a solution of ester $\mathbf{1}(3.83 \mathrm{~g}, 12.1 \mathrm{mmol})$ in $\operatorname{dry}^{\mathrm{Et}} \mathrm{C}_{2} \mathrm{O}(70 \mathrm{~mL})$. The reaction mixture was stirred at $0{ }^{\circ} \mathrm{C}$ for $15 \mathrm{~min}$ and then for $45 \mathrm{~min}$ at room temperature. The reaction 
was quenched by careful addition of water $(3 \mathrm{~mL})$ and the precipitate was removed by filtration. The filtrate was dried $\left(\mathrm{Na}_{2} \mathrm{SO}_{4}\right)$ and concentrated under reduced pressure. The chromatography of the residue on silica gel (hexane-ethyl acetate, 2:1) afforded $3.03 \mathrm{~g}(88 \%)$ of alcohol 3 as a colorless oil; $[\alpha]_{\mathrm{D}}{ }^{25}=-23\left(c 0.49, \mathrm{CHCl}_{3}\right) ;{ }^{1} \mathrm{H}-\mathrm{NMR}: \delta 1.34\left(3 \mathrm{H}, \mathrm{s}, \mathrm{CH}_{3}\right), 1.38\left(3 \mathrm{H}, \mathrm{s}, \mathrm{CH}_{3}\right), 1.44\left(3 \mathrm{H}, \mathrm{s}, \mathrm{CH}_{3}\right), 1.48$ $\left(3 \mathrm{H}, \mathrm{s}, \mathrm{CH}_{3}\right), 1.93\left(1 \mathrm{H}, \mathrm{m}, \mathrm{H}_{2}\right), 2.05\left(1 \mathrm{H}, \mathrm{m}, \mathrm{H}_{2}\right), 3.54\left(1 \mathrm{H}, \mathrm{dd}, J_{7,6}=7.2 \mathrm{~Hz}, J_{6,5}=3.7 \mathrm{~Hz}, \mathrm{H}_{6}\right), 3.70(1 \mathrm{H}$, ddd, $\left.J_{3,2}=8.4 \mathrm{~Hz}, J_{3,2}=5.1 \mathrm{~Hz}, J_{4,3}=3.7 \mathrm{~Hz}, \mathrm{H}_{3}\right), 3.80\left(2 \mathrm{H}, \mathrm{m}, \mathrm{H}_{1}\right), 4.05\left(1 \mathrm{H}, \mathrm{dd}, J_{8,8}=8.7 \mathrm{~Hz}, J_{8,7}=4.8\right.$ $\left.\mathrm{Hz}, \mathrm{H}_{8}\right), 4.09\left(1 \mathrm{H}, \mathrm{dd}, J_{8,8}=8.7 \mathrm{~Hz}, J_{8,7}=6.1 \mathrm{~Hz}, \mathrm{H}_{8}\right), 4.40\left(1 \mathrm{H}, \mathrm{m}, \mathrm{H}_{7}\right), 4.66\left(1 \mathrm{H}, \mathrm{dd}, J_{5,4}=6.1 \mathrm{~Hz}\right.$, $\left.J_{4,3}=3.7 \mathrm{~Hz}, \mathrm{H}_{4}\right), 4.76\left(1 \mathrm{H}, \mathrm{dd}, J_{5,4}=6.1 \mathrm{~Hz}, J_{6,5}=3.7 \mathrm{~Hz}, \mathrm{H}_{5}\right) ;{ }^{13} \mathrm{C}-\mathrm{NMR}: \delta 24.6,25.3,25.7,26.9,31.1$, 60.4, 66.8, 73.1, 80.5, 80.6, 81.7, 81.7, 109.0, 112.4; Anal. Calcd for $\mathrm{C}_{14} \mathrm{H}_{24} \mathrm{O}_{6}$ (288.34): C 58.32, $\mathrm{H}$ 8.39; found C 58.54, H 8.66.

3,6-Anhydro-2-deoxy-4,5:7,8-di-O-isopropylidene-D-glycero-D-galacto-octose (4): To a solution of alcohol 3 (3.0 g, $10.5 \mathrm{mmol})$ in $\mathrm{CH}_{3} \mathrm{CN}(55 \mathrm{~mL})$ was added IBX (4.41 g, $\left.15.7 \mathrm{mmol}\right)$. The resulting suspension was heated under reflux for $40 \mathrm{~min}$. Then the reaction was cooled to room temperature and filtered through a medium glass frit. The filter cake was washed with further portions of acetonitrile ( 2 x $15 \mathrm{~mL}$ ). The combined filtrates were concentrated under reduced pressure. The chromatography of the residue on silica gel (hexane-ethyl acetate, 3:1) afforded $2.73 \mathrm{~g}(93 \%)$ of aldehyde 4 as a colorless oil; $[\alpha]_{\mathrm{D}}{ }^{25}=-12\left(c 0.68, \mathrm{CHCl}_{3}\right) ;{ }^{1} \mathrm{H}-\mathrm{NMR}: \delta 1.32\left(3 \mathrm{H}, \mathrm{s}, \mathrm{CH}_{3}\right), 1.38\left(3 \mathrm{H}, \mathrm{s}, \mathrm{CH}_{3}\right), 1.45\left(3 \mathrm{H}, \mathrm{s}, \mathrm{CH}_{3}\right)$, $1.46\left(3 \mathrm{H}, \mathrm{s}, \mathrm{CH}_{3}\right), 2.87-2.89\left(2 \mathrm{H}, \mathrm{m}, \mathrm{H}_{2}\right), 3.55\left(1 \mathrm{H}, \mathrm{dd}, J_{7,6}=7.4 \mathrm{~Hz}, J_{6,5}=3.3 \mathrm{~Hz}, \mathrm{H}_{6}\right), 3.99(1 \mathrm{H}$, ddd, $\left.J_{3,2}=6.4 \mathrm{~Hz}, J_{3,2}=6.4 \mathrm{~Hz}, J_{4,3}=3.3 \mathrm{~Hz}, \mathrm{H}_{3}\right), 4.03\left(1 \mathrm{H}, \mathrm{dd}, J_{8,8}=8.7 \mathrm{~Hz}, J_{8,7}=4.7 \mathrm{~Hz}, \mathrm{H}_{8}\right), 4.08(1 \mathrm{H}, \mathrm{dd}$, $\left.J_{8,8}=8.7 \mathrm{~Hz}, J_{8,7}=6.2 \mathrm{~Hz}, \mathrm{H}_{8}\right), 4.39\left(1 \mathrm{H}, \mathrm{ddd}, J_{7,6}=7.4 \mathrm{~Hz}, J_{8,7}=6.2 \mathrm{~Hz}, J_{8,7}=4.7 \mathrm{~Hz}, \mathrm{H}_{7}\right), 4.76(1 \mathrm{H}, \mathrm{dd}$, $\left.J_{5,4}=6.1 \mathrm{~Hz}, J_{4,3}=3.3 \mathrm{~Hz}, \mathrm{H}_{4}\right), 4.79\left(1 \mathrm{H}, \mathrm{dd}, J_{5,4}=6.1 \mathrm{~Hz}, J_{6,5}=3.3 \mathrm{~Hz}, \mathrm{H}_{5}\right), 9.81(1 \mathrm{H}, \mathrm{t}, J=1.3 \mathrm{~Hz}, \mathrm{CHO})$; ${ }^{13} \mathrm{C}-\mathrm{NMR}: \delta$ 24.5, 25.2, 25.6, 26.9, 42.8, 66.8, 73.0, 76.6, 80.6, 81.0, 81.6, 109.1, 112.6, 199.9; Anal. Calcd for $\mathrm{C}_{14} \mathrm{H}_{22} \mathrm{O}_{6}$ (286.33): C 58.73, H 7.74; found C 58.49, H 7.51.

Methyl 5,8-anhydro-2,3,4-trideoxy-6,7:9,10-di-O-isopropylidene-D-glycero-D-galacto-dec-2(E)enoate (5): [(Methoxycarbonyl)methylidene]triphenylphosphorane (3.82 g, $11.4 \mathrm{mmol})$ was added to a solution of aldehyde $4(2.73 \mathrm{~g}, 9.5 \mathrm{mmol})$ in dry $\mathrm{CH}_{2} \mathrm{Cl}_{2}(25 \mathrm{~mL})$. The reaction mixture was stirred for $1.5 \mathrm{~h}$ at room temperature. The solvent was removed under reduced pressure and the residue was purified by chromatography on silica gel (hexane-ethyl acetate, 5:1) to afford $2.79 \mathrm{~g}(87 \%)$ of $(E)-5$ as a colorless oil; $[\alpha]_{\mathrm{D}}{ }^{25}=-12\left(c 0.34, \mathrm{CHCl}_{3}\right) ;{ }^{1} \mathrm{H}-\mathrm{NMR}: \delta 1.33\left(3 \mathrm{H}, \mathrm{s}, \mathrm{CH}_{3}\right), 1.38\left(3 \mathrm{H}, \mathrm{s}, \mathrm{CH}_{3}\right), 1.44$ $\left(3 \mathrm{H}, \mathrm{s}, \mathrm{CH}_{3}\right), 1.48\left(3 \mathrm{H}, \mathrm{s}, \mathrm{CH}_{3}\right), 2.61\left(2 \mathrm{H}, \mathrm{m}, \mathrm{H}_{4}\right), 3.50\left(1 \mathrm{H}, \mathrm{dd}, J_{9,8}=7.5 \mathrm{~Hz}, J_{8,7}=3.6 \mathrm{~Hz}, \mathrm{H}_{8}\right), 3.59$ $\left(1 \mathrm{H}\right.$, ddd, $\left.J_{5,4}=6.8 \mathrm{~Hz}, J_{5,4}=6.8 \mathrm{~Hz}, J_{6,5}=3.6 \mathrm{~Hz}, \mathrm{H}_{5}\right), 3.73\left(3 \mathrm{H}, \mathrm{s}, \mathrm{OCH}_{3}\right), 4.05\left(1 \mathrm{H}, \mathrm{dd}, J_{10,10}=8.7 \mathrm{~Hz}\right.$, $\left.J_{10,9}=4.8 \mathrm{~Hz}, \mathrm{H}_{10}\right), 4.09\left(1 \mathrm{H}, \mathrm{dd}, J_{10,10}=8.7 \mathrm{~Hz}, J_{10,9}=6.1 \mathrm{~Hz}, \mathrm{H}_{10}\right), 4.39\left(1 \mathrm{H}, \mathrm{ddd}, J_{9,8}=7.5 \mathrm{~Hz}, J_{10,9}=6.1\right.$ $\left.\mathrm{Hz}, J_{10,9}=4.8 \mathrm{~Hz}, \mathrm{H}_{9}\right), 4.63\left(1 \mathrm{H}, \mathrm{dd}, J_{7,6}=6.1 \mathrm{~Hz}, J_{6,5}=3.6 \mathrm{~Hz}, \mathrm{H}_{6}\right), 4.76\left(1 \mathrm{H}, \mathrm{dd}, J_{7,6}=6.1 \mathrm{~Hz}, J_{8,7}=3.6\right.$ $\left.\mathrm{Hz}, \mathrm{H}_{7}\right), 5.94\left(1 \mathrm{H}\right.$, ddd, $\left.J_{3,2}=15.7 \mathrm{~Hz}, J_{4,2}=1.5 \mathrm{~Hz}, J_{4,2}=1.5 \mathrm{~Hz}, \mathrm{H}_{2}\right), 6.99\left(1 \mathrm{H}\right.$, ddd, $J_{3,2}=15.7 \mathrm{~Hz}$, $\left.J_{4,3}=7.1 \mathrm{~Hz}, J_{4,3}=7.1 \mathrm{~Hz}, \mathrm{H}_{3}\right) ;{ }^{13} \mathrm{C}-\mathrm{NMR}: \delta 24.6,25.3,25.7,26.9,31.4,51.5,66.9,73.1,80.3,80.7$, 81.1, 81.7, 109.1, 112.6, 123.0, 144.9, 166.8; Anal. Calcd for $\mathrm{C}_{17} \mathrm{H}_{26} \mathrm{O}_{7}$ (342.39): C 59.64, $\mathrm{H}$ 7.65; found C 59.73, H 7.79 .

5,8-Anhydro-2,3,4-trideoxy-6, 7:9,10-di-O-isopropylidene-D-glycero-D-galacto-dec-2(E)-enitol (6): To a solution of ester $5(2.79 \mathrm{~g}, 8.15 \mathrm{mmol})$ in dry $\mathrm{CH}_{2} \mathrm{Cl}_{2}(37 \mathrm{~mL})$ diisobutylaluminum hydride $(24.6 \mathrm{~mL}$ of 
1.2 $\mathrm{M}$ toluene solution) was added dropwise at $-15^{\circ} \mathrm{C}$. The resulting mixture was stirred for $45 \mathrm{~min}$ at the same temperature and then quenched with methanol $(6.2 \mathrm{~mL})$. The mixture was allowed to warm to room temperature and poured into $30 \%$ aqueous $\mathrm{K} / \mathrm{Na}$ tartrate $(123 \mathrm{~mL})$. After stirring for $30 \mathrm{~min}$, the product was extracted with $\mathrm{CH}_{2} \mathrm{Cl}_{2}(3 \times 37 \mathrm{~mL})$. The combined organic layers were dried $\left(\mathrm{Na}_{2} \mathrm{SO}_{4}\right)$ and the solvent evaporated under reduced pressure. Chromatography of the residue on silica gel (hexane-ethyl acetate, $1: 1)$ afforded $2.34 \mathrm{~g}(91 \%)$ of allylic alcohol 6 as a colorless oil; $[\alpha]_{\mathrm{D}}{ }^{25}=+22$ (c $\left.0.28, \mathrm{CHCl}_{3}\right)$; ${ }^{1} \mathrm{H}-\mathrm{NMR}: \delta 1.34\left(3 \mathrm{H}, \mathrm{s}, \mathrm{CH}_{3}\right), 1.38\left(3 \mathrm{H}, \mathrm{s}, \mathrm{CH}_{3}\right), 1.45\left(3 \mathrm{H}, \mathrm{s}, \mathrm{CH}_{3}\right), 1.48(3 \mathrm{H}, \mathrm{s}$, $\left.\mathrm{CH}_{3}\right), 2.47\left(2 \mathrm{H}, \mathrm{m}, \mathrm{H}_{4}\right), 3.48\left(1 \mathrm{H}, \mathrm{m}, \mathrm{H}_{8}\right), 3.52\left(1 \mathrm{H}, \mathrm{m}, \mathrm{H}_{5}\right), 4.05\left(1 \mathrm{H}, \mathrm{dd}, J_{10,10}=8.7 \mathrm{~Hz}, J_{10,9}=4.8 \mathrm{~Hz}\right.$, $\left.\mathrm{H}_{10}\right), 4.08\left(1 \mathrm{H}, \mathrm{dd}, J_{10,10}=8.7 \mathrm{~Hz}, J_{10,9}=6.0 \mathrm{~Hz}, \mathrm{H}_{10}\right), 4.11\left(2 \mathrm{H}, \mathrm{m}, \mathrm{H}_{1}\right), 4.40\left(1 \mathrm{H}, \mathrm{ddd}, J_{9,8}=7.4 \mathrm{~Hz}\right.$, $\left.J_{10,9}=6.0 \mathrm{~Hz}, J_{10,9}=4.8 \mathrm{~Hz}, \mathrm{H}_{9}\right), 4.62\left(1 \mathrm{H}, \mathrm{dd}, J_{7,6}=6.1 \mathrm{~Hz}, J_{6,5}=3.6 \mathrm{~Hz}, \mathrm{H}_{6}\right), 4.74\left(1 \mathrm{H}, \mathrm{dd}, J_{7,6}=6.1 \mathrm{~Hz}\right.$, $\left.J_{8,7}=3.7 \mathrm{~Hz}, \mathrm{H}_{7}\right), 5.76\left(2 \mathrm{H}, \mathrm{m}, \mathrm{H}_{2}, \mathrm{H}_{3}\right) ;{ }^{13} \mathrm{C}-\mathrm{NMR}: \delta 24.7,25.3,25.8,26.9,31.2,63.6,66.9,73.2,80.7$, 81.1, 81.5, 81.6, 109.0, 112.4, 128.2, 131.5; Anal. Calcd for $\mathrm{C}_{16} \mathrm{H}_{26} \mathrm{O}_{6}$ (314.38): C 61.13, H 8.34; found C 61.32, H 8.50.

5,8-Anhydro-1,2,3,4-tetradeoxy-6,7:9,10-di-O-isopropylidene-1-thiocyanato-D-glycero-D-galactodec-2(E)-enitol (7): To a solution of alcohol 6 (2.34 g, $7.44 \mathrm{mmol})$ in dry dichloromethane $(26 \mathrm{~mL})$ were added triethylamine $(1.55 \mathrm{~mL}, 11.17 \mathrm{mmol})$ and $\mathrm{CH}_{3} \mathrm{SO}_{2} \mathrm{Cl}(0.69 \mathrm{~mL}, 8.93 \mathrm{mmol})$ at $0{ }^{\circ} \mathrm{C}$. The mixture was stirred at $0{ }^{\circ} \mathrm{C}$ for $15 \mathrm{~min}$ and then further $45 \mathrm{~min}$ at room temperature. The solvent was evaporated under reduced pressure. The residue was diluted with diethyl ether $(40 \mathrm{~mL})$ and the solid was removed by filtration. The solvent was evaporated to afford the crude mesylate which was used in the subsequent reaction directly without further purification. To the crude mesylate dissolved in $\mathrm{CH}_{3} \mathrm{CN}(26 \mathrm{~mL}), \mathrm{KSCN}(1.09 \mathrm{~g}, 11.17 \mathrm{mmol})$ was added. After stirring at room temperature for $1 \mathrm{~h}$, the solvent was evaporated. The residue was diluted with diethyl ether $(40 \mathrm{~mL})$ and the solid was removed by filtration. Evaporation of the solvent and chromatography of the residue (hexane-ethyl acetate, $5: 1)$ afforded $2.0 \mathrm{~g}(76 \%)$ of thiocyanate 7 as white crystals; m.p. $81-82{ }^{\circ} \mathrm{C} ;[\alpha]_{\mathrm{D}}{ }^{25}=+23(c$ 0.28, $\left.\mathrm{CHCl}_{3}\right)$; ${ }^{1} \mathrm{H}-\mathrm{NMR}: \delta 1.34\left(3 \mathrm{H}, \mathrm{s}, \mathrm{CH}_{3}\right), 1.38\left(3 \mathrm{H}, \mathrm{s}, \mathrm{CH}_{3}\right), 1.44\left(3 \mathrm{H}, \mathrm{s}, \mathrm{CH}_{3}\right), 1.48\left(3 \mathrm{H}, \mathrm{s}, \mathrm{CH}_{3}\right)$, 2.50-2.54 (2H, m, $\left.\mathrm{H}_{4}\right), 3.49\left(1 \mathrm{H}, \mathrm{dd}, J_{9,8}=7.5 \mathrm{~Hz}, J_{8,7}=3.7 \mathrm{~Hz}, \mathrm{H}_{8}\right), 3.52-3.55\left(3 \mathrm{H}, \mathrm{m}, 2 \mathrm{x} \mathrm{H}_{1}, \mathrm{H}_{5}\right), 4.05$ $\left(1 \mathrm{H}, \mathrm{dd}, J_{10,10}=8.7 \mathrm{~Hz}, J_{10,9}=4.8 \mathrm{~Hz}, \mathrm{H}_{10}\right), 4.09\left(1 \mathrm{H}, \mathrm{dd}, J_{10,10}=8.7 \mathrm{~Hz}, J_{10,9}=6.1 \mathrm{~Hz}, \mathrm{H}_{10}\right), 4.39(1 \mathrm{H}$, ddd, $\left.J_{9,8}=7.5 \mathrm{~Hz}, J_{10,9}=6.1 \mathrm{~Hz}, J_{10,9}=4.8 \mathrm{~Hz}, \mathrm{H}_{9}\right), 4.67\left(1 \mathrm{H}, \mathrm{dd}, J_{7,6}=6.1 \mathrm{~Hz}, J_{6,5}=3.6 \mathrm{~Hz}, \mathrm{H}_{6}\right), 4.74(1 \mathrm{H}$, $\left.\mathrm{dd}, J_{7,6}=6.1 \mathrm{~Hz}, J_{8,7}=3.7 \mathrm{~Hz}, \mathrm{H}_{7}\right), 5.70\left(1 \mathrm{H}, \mathrm{m}, \mathrm{H}_{2}\right), 5.87\left(1 \mathrm{H}, \mathrm{m}, \mathrm{H}_{3}\right) ;{ }^{13} \mathrm{C}-\mathrm{NMR}: \delta 24.6,25.3,25.8$, 26.9, 31.4, 36.3, 66.9, 73.1, 80.7 81.0, 81.2, 81.6, 109.0, 111.9, 112.4, 124.9, 134.0; Anal. Calcd for $\mathrm{C}_{17} \mathrm{H}_{25} \mathrm{NO}_{5} \mathrm{~S}$ (355.46): C 57.44, H 7.09, N 3.94; found C 57.61, H 7.28, N 4.04.

5,8-Anhydro-1,2,3,4-tetradeoxy-6,7:9,10-di-O-isopropylidene-3(S)-isothiocyanato-D-glycero-Dgalacto-dec-1-enitol (8a) and 5,8-Anhydro-1,2,3,4-tetradeoxy-6,7:9,10-di-O-isopropylidene-3(R)isothiocyanato-D-glycero-D-galacto-dec-1-enitol (8b): Conventional method for the preparation of $\mathbf{8 a}$, 8b: A solution of thiocyanate $7(1.80 \mathrm{~g}, 5.06 \mathrm{mmol})$ in dry $n$-heptane $(30 \mathrm{~mL})$ was heated at $90{ }^{\circ} \mathrm{C}$ for $6 \mathrm{~h}$ under nitrogen atmosphere. The solvent was evaporated under reduced pressure. The chromatography of the residue on silica gel (hexane-ethyl acetate, 9:1) afforded isothiocyanates 8a $(0.75 \mathrm{~g}, 42 \%)$ and $\mathbf{8 b}(0.73 \mathrm{~g}, 41 \%)$. 
Microwave-assisted synthesis of $\mathbf{8 a}, \mathbf{8 b}$ : The $(E)$-thiocyanate $7(20 \mathrm{mg}, 0.056 \mathrm{mmol})$ was weighed in a $10 \mathrm{ml}$ glass pressure microwave tube equipped with a magnetic stirrer bar. Dry $n$-heptane $(0.4 \mathrm{~mL})$ was added, the tube was closed with a silicon septum and the reaction mixture was subjected to microwave irradiation for $2 \mathrm{~h}$ (power: $150 \mathrm{~W}$, temperature: $90{ }^{\circ} \mathrm{C}$, pressure: $12 \mathrm{bar}$ ). The reaction mixture was allowed to cool to room temperature and transferred into a round bottom flask. The solvent was evaporated under reduced pressure. The chromatography of the residue on silica gel (hexane-ethyl acetate, 9:1) gave $0.16 \mathrm{mg}(86 \%)$ of isothiocyanates 8a, 8b. Compound 8a: white crystals; m.p. $54-56{ }^{\circ} \mathrm{C} ;[\alpha]_{\mathrm{D}}{ }^{25}=-19\left(c 0.27, \mathrm{CHCl}_{3}\right)$; $v_{\max }$ (liquid film) 2033 (NCS) cm ${ }^{-1} ;{ }^{1} \mathrm{H}-\mathrm{NMR}: \delta$ $1.33\left(3 \mathrm{H}, \mathrm{s}, \mathrm{CH}_{3}\right), 1.38\left(3 \mathrm{H}, \mathrm{s}, \mathrm{CH}_{3}\right), 1.45\left(3 \mathrm{H}, \mathrm{s}, \mathrm{CH}_{3}\right), 1.46\left(3 \mathrm{H}, \mathrm{s}, \mathrm{CH}_{3}\right), 1.93\left(1 \mathrm{H}, \mathrm{m}, \mathrm{H}_{4}\right), 2.07(1 \mathrm{H}$, ddd, $\left.J_{4,4}=14.3 \mathrm{~Hz}, J_{5,4}=9.3 \mathrm{~Hz}, J_{4,3}=3.7 \mathrm{~Hz}, \mathrm{H}_{4}\right), 3.54\left(1 \mathrm{H}, \mathrm{dd}, J_{9,8}=7.6 \mathrm{~Hz}, J_{8,7}=3.7 \mathrm{~Hz}, \mathrm{H}_{8}\right), 3.71(1 \mathrm{H}$, ddd, $\left.J_{5,4}=9.3 \mathrm{~Hz}, J_{6,5}=3.7 \mathrm{~Hz}, J_{5,4}=3.5 \mathrm{~Hz}, \mathrm{H}_{5}\right), 4.06\left(1 \mathrm{H}, \mathrm{dd}, J_{10,10}=8.7 \mathrm{~Hz}, J_{10,9}=4.8 \mathrm{~Hz}, \mathrm{H}_{10}\right), 4.09$ $\left(1 \mathrm{H}, \mathrm{dd}, J_{10,10}=8.7 \mathrm{~Hz}, J_{10,9}=6.0 \mathrm{~Hz}, \mathrm{H}_{10}\right), 4.39\left(1 \mathrm{H}, \mathrm{ddd}, J_{9,8}=7.6 \mathrm{~Hz}, J_{10,9}=6.0 \mathrm{~Hz}, J_{10,9}=4.8 \mathrm{~Hz}, \mathrm{H}_{9}\right)$, $4.45\left(1 \mathrm{H}, \mathrm{m}, \mathrm{H}_{3}\right), 4.64\left(1 \mathrm{H}, \mathrm{dd}, J_{7,6}=6.1 \mathrm{~Hz}, J_{6,5}=3.7 \mathrm{~Hz}, \mathrm{H}_{6}\right), 4.78\left(1 \mathrm{H}, \mathrm{dd}, J_{7,6}=6.1 \mathrm{~Hz}, J_{8,7}=3.7 \mathrm{~Hz}\right.$, $\left.\mathrm{H}_{7}\right), 5.24\left(1 \mathrm{H}, \mathrm{dd}, J_{2,1 \text { cis }}=10.2 \mathrm{~Hz}, J_{3,1 \text { cis }}=1.4 \mathrm{~Hz}, \mathrm{H}_{1 \text { cis }}\right), 5.39\left(1 \mathrm{H}, \mathrm{dd}, J_{2,1 \text { trans }}=16.9 \mathrm{~Hz}, J_{3,1 \text { trans }}=1.6 \mathrm{~Hz}\right.$, $\left.\mathrm{H}_{1 \text { trans }}\right), 5.83\left(1 \mathrm{H}\right.$, ddd, $\left.J_{2,1 \text { trans }}=16.9 \mathrm{~Hz}, J_{2,1 \text { cis }}=10.2 \mathrm{~Hz}, J_{3,2}=5.4 \mathrm{~Hz}, \mathrm{H}_{2}\right) ;{ }^{13} \mathrm{C}-\mathrm{NMR}: \delta 24.5,25.3,25.7$, 26.9, 35.3, 57.5, 66.9, 73.0, 78.1, 80.8, 81.5, 81.7, 109.2, 112.5, 116.5, 132.9, 135.2; Anal. Calcd for $\mathrm{C}_{17} \mathrm{H}_{25} \mathrm{NO}_{5} \mathrm{~S}$ (355.46): C 57.44, H 7.09, N 3.94; found C 57.25, H 7.20, N 4.11. Compound 8b: a colorless oil; $[\alpha]_{\mathrm{D}}{ }^{25}=-10\left(c 0.41, \mathrm{CHCl}_{3}\right) ; v_{\max }$ (liquid film) 2020 (NCS) cm ${ }^{-1} ;{ }^{1} \mathrm{H}-\mathrm{NMR}: \delta 1.33(3 \mathrm{H}, \mathrm{s}$, $\left.\mathrm{CH}_{3}\right), 1.38\left(3 \mathrm{H}, \mathrm{s}, \mathrm{CH}_{3}\right), 1.44\left(3 \mathrm{H}, \mathrm{s}, \mathrm{CH}_{3}\right), 1.47\left(3 \mathrm{H}, \mathrm{s}, \mathrm{CH}_{3}\right), 2.03\left(1 \mathrm{H}, \mathrm{m}, \mathrm{H}_{4}\right), 2.16\left(1 \mathrm{H}, \mathrm{m}, \mathrm{H}_{4}\right), 3.49$ $\left(1 \mathrm{H}, \mathrm{dd}, J_{9,8}=7.6 \mathrm{~Hz}, J_{8,7}=3.6 \mathrm{~Hz}, \mathrm{H}_{8}\right), 3.61\left(1 \mathrm{H}\right.$, ddd, $\left.J_{5,4}=7.4 \mathrm{~Hz}, J_{5,4}=6.1 \mathrm{~Hz}, J_{6,5}=3.7 \mathrm{~Hz}, \mathrm{H}_{5}\right), 4.05$ $\left(1 \mathrm{H}, \mathrm{dd}, J_{10,10}=8.8 \mathrm{~Hz}, J_{10,9}=4.6 \mathrm{~Hz}, \mathrm{H}_{10}\right), 4.09\left(1 \mathrm{H}, \mathrm{dd}, J_{10,10}=8.8 \mathrm{~Hz}, J_{10,9}=6.2 \mathrm{~Hz}, \mathrm{H}_{10}\right), 4.36-4.41$ $\left(2 \mathrm{H}, \mathrm{m}, \mathrm{H}_{3}, \mathrm{H}_{9}\right), 4.64\left(1 \mathrm{H}, \mathrm{dd}, J_{7,6}=6.1 \mathrm{~Hz}, J_{6,5}=3.7 \mathrm{~Hz}, \mathrm{H}_{6}\right), 4.77\left(1 \mathrm{H}, \mathrm{dd}, J_{7,6}=6.1 \mathrm{~Hz}, J_{8,7}=3.6 \mathrm{~Hz}\right.$, $\left.\mathrm{H}_{7}\right), 5.25\left(1 \mathrm{H}, \mathrm{ddd}, J_{2,1^{\prime} c i s}=10.2 \mathrm{~Hz}, J_{3,1 \text { cis }}=1.1 \mathrm{~Hz}, J_{1 \text { cis }, 1 \text { trans }}=0.5 \mathrm{~Hz}, \mathrm{H}_{1 \text { cis }}\right), 5.36\left(1 \mathrm{H}, \mathrm{ddd}, J_{2,1 \text { trans }}=16.8\right.$ $\left.\mathrm{Hz}, J_{3,1 \text { trans }}=1.3 \mathrm{~Hz}, J_{1 \text { cis, } 1 \text { trans }}=0.5 \mathrm{~Hz}, \mathrm{H}_{1 \text { trans }}\right), 5.81\left(1 \mathrm{H}\right.$, ddd, $J_{2,1 \text { trans }}=16.8 \mathrm{~Hz}, J_{2,1 \text { cis }}=10.2 \mathrm{~Hz}, J_{3,2}=6.0$ $\left.\mathrm{Hz}, \mathrm{H}_{2}\right) ;{ }^{13} \mathrm{C}-\mathrm{NMR}: \delta 24.6,25.2,25.7,27.0,34.8,57.3,66.9,73.0,78.4,80.7,81.0,81.7,109.1,112.6$, 117.2, 132.7, 134.8; Anal. Calcd for $\mathrm{C}_{17} \mathrm{H}_{25} \mathrm{NO}_{5} \mathrm{~S}$ (355.46): C 57.44, H 7.09, N 3.94; found C 57.62, $\mathrm{H}$ 6.94, N 3.88 .

\section{5,8-Anhydro-1,2,3,4-tetradeoxy-6,7:9,10-di-O-isopropylidene-3(S)-(methoxycarbonylamino)-D-}

glycero-D-galacto-dec-1-enitol (10a): To a solution of isothiocyanate $\mathbf{8 a}(0.54 \mathrm{~g}, 1.52 \mathrm{mmol})$ in dry methanol $(15 \mathrm{~mL})$ was added sodium methoxide $(90 \mathrm{mg}, 1.67 \mathrm{mmol})$. The reaction mixture was stirred for $3 \mathrm{~h}$ at room temperature under nitrogen atmosphere. The solvent was evaporated and the residue was partitioned between $\mathrm{CH}_{2} \mathrm{Cl}_{2}(25 \mathrm{~mL})$ and water $(7 \mathrm{~mL})$. The organic layer was dried $\left(\mathrm{Na}_{2} \mathrm{SO}_{4}\right)$ and the solvent was evaporated under reduced pressure to provide the crude thiourethane 9a which was used in the subsequent reaction directly without further purification. To a solution of 9a (436 mg, 1.12 $\mathrm{mmol})$ in dry acetonitrile $(10.8 \mathrm{~mL})$ was added mesitonitrile oxide $(218 \mathrm{mg}, 1.35 \mathrm{mmol})$. The mixture was stirred at room temperature for $2 \mathrm{~h}$ under nitrogen atmosphere. The solvent was evaporated under reduced pressure. The chromatography of the residue (hexane-ethyl acetate, $3: 1$ ) gave $0.35 \mathrm{~g}(85 \%)$ of 10a as a colorless oil; ${ }^{1} \mathrm{H}-\mathrm{NMR}: \delta 1.33\left(3 \mathrm{H}, \mathrm{s}, \mathrm{CH}_{3}\right), 1.38\left(3 \mathrm{H}, \mathrm{s}, \mathrm{CH}_{3}\right), 1.45\left(3 \mathrm{H}, \mathrm{s}, \mathrm{CH}_{3}\right), 1.47(3 \mathrm{H}, \mathrm{s}$, $\left.\mathrm{CH}_{3}\right), 1.88\left(1 \mathrm{H}, \mathrm{m}, \mathrm{H}_{4}\right), 2.04\left(1 \mathrm{H}, \mathrm{m}, \mathrm{H}_{4}\right), 3.51\left(1 \mathrm{H}, \mathrm{dd}, J_{9,8}=6.7 \mathrm{~Hz}, J_{8,7}=3.7 \mathrm{~Hz}, \mathrm{H}_{8}\right), 3.63(1 \mathrm{H}, \mathrm{m}$, $\left.\mathrm{H}_{5}\right), 3.66\left(3 \mathrm{H}, \mathrm{s}, \mathrm{CH}_{3} \mathrm{O}\right), 4.05\left(1 \mathrm{H}, \mathrm{dd}, J_{10,10}=8.7 \mathrm{~Hz}, J_{10,9}=4.8 \mathrm{~Hz}, \mathrm{H}_{10}\right), 4.10\left(1 \mathrm{H}, \mathrm{dd}, J_{10,10}=8.7 \mathrm{~Hz}\right.$, $\left.J_{10,9}=6.2 \mathrm{~Hz}, \mathrm{H}_{10}\right), 4.36-4.41\left(2 \mathrm{H}, \mathrm{m}, \mathrm{H}_{3}, \mathrm{H}_{9}\right), 4.59\left(1 \mathrm{H}, \mathrm{dd}, J_{7,6}=6.1 \mathrm{~Hz}, J_{6,5}=3.7 \mathrm{~Hz}, \mathrm{H}_{6}\right), 4.72(1 \mathrm{H}, \mathrm{dd}$, 
$\left.J_{7,6}=6.1 \mathrm{~Hz}, J_{8,7}=3.7 \mathrm{~Hz}, \mathrm{H}_{7}\right), 5.13\left(1 \mathrm{H}, \mathrm{d}, J_{2,1 c i s}=10.4 \mathrm{~Hz}, \mathrm{H}_{1 c i s}\right), 5.20\left(1 \mathrm{H}, \mathrm{d}, J_{2,1 \text { trans }}=17.1 \mathrm{~Hz}, \mathrm{H}_{1 \text { trans }}\right)$, $5.38\left(1 \mathrm{H}, \mathrm{d}, J_{3, \mathrm{NH}}=6.4 \mathrm{~Hz}, \mathrm{NH}\right), 5.80\left(1 \mathrm{H}, \mathrm{ddd}, J_{2,1 \text { trans }}=17.1 \mathrm{~Hz}, J_{2,1 \text { cis }}=10.4 \mathrm{~Hz}, J_{3,2}=5.1 \mathrm{~Hz}, \mathrm{H}_{2}\right) ;{ }^{13} \mathrm{C}$ NMR: $\delta$ 24.5, 25.3, 25.7, 26.9, 32.6, 51.0, 52.0, 66.8, 73.1, 79.1, 80.5, 81.7, 81.8, 109.1, 112.5, 114.7, 138.2, 156.4; Anal. Calcd for $\mathrm{C}_{18} \mathrm{H}_{29} \mathrm{NO}_{7}$ (371.43): C 58.21, H 7.87, N 3.77; found C 58.46, H 7.61, N 3.92 .

\section{5,8-Anhydro-1,2,3,4-tetradeoxy-6,7:9,10-di-O-isopropylidene-3(R)-(methoxycarbonylamino)-D-} glycero-D-galacto-dec-1-enitol (10b): To a solution of isothiocyanate $\mathbf{8 b}(416 \mathrm{mg}, 1.17 \mathrm{mmol})$ in dry methanol $(11.6 \mathrm{~mL})$ was added sodium methoxide $(69.5 \mathrm{mg}, 1.29 \mathrm{mmol})$. The reaction mixture was stirred for $4 \mathrm{~h}$ at room temperature under nitrogen atmosphere. The solvent was evaporated and the residue was partitioned between $\mathrm{CH}_{2} \mathrm{Cl}_{2}(20 \mathrm{~mL})$ and water $(6 \mathrm{~mL})$. The organic layer was dried $\left(\mathrm{Na}_{2} \mathrm{SO}_{4}\right)$. The solvent was evaporated under reduced pressure to give the crude thiourethane $\mathbf{9 b}$ which was used in the subsequent reaction directly without further purification. To a solution of $\mathbf{9 b}(288 \mathrm{mg}$, $0.74 \mathrm{mmol})$ in dry acetonitrile $(7 \mathrm{~mL})$ was added mesitonitrile oxide (144 mg, $0.89 \mathrm{mmol})$. The mixture was stirred at room temperature for $2 \mathrm{~h}$ under nitrogen atmosphere. The solvent was evaporated under reduced pressure and the chromatography of the residue (hexane-ethyl acetate, $3: 1$ ) afforded $0.25 \mathrm{~g}(92 \%)$ of $\mathbf{1 0 b}$ as a colorless oil; ${ }^{1} \mathrm{H}-\mathrm{NMR}: \delta 1.33\left(3 \mathrm{H}, \mathrm{s}, \mathrm{CH}_{3}\right), 1.37\left(3 \mathrm{H}, \mathrm{s}, \mathrm{CH}_{3}\right), 1.44$ $\left(3 \mathrm{H}, \mathrm{s}, \mathrm{CH}_{3}\right), 1.47\left(3 \mathrm{H}, \mathrm{s}, \mathrm{CH}_{3}\right), 1.84\left(1 \mathrm{H}, \mathrm{ddd}, J_{4,4}=14.2 \mathrm{~Hz}, J_{4,3}=9.5 \mathrm{~Hz}, J_{5,4}=6.5 \mathrm{~Hz}, \mathrm{H}_{4}\right), 2.00(1 \mathrm{H}$, $\left.\mathrm{m}, \mathrm{H}_{4}\right), 3.47\left(1 \mathrm{H}, \mathrm{dd}, J_{9,8}=7.5 \mathrm{~Hz}, J_{8,7}=3.6 \mathrm{~Hz}, \mathrm{H}_{8}\right), 3.59\left(1 \mathrm{H}\right.$, ddd, $J_{5,4}=6.5 \mathrm{~Hz}, J_{5,4}=6.5 \mathrm{~Hz}, J_{6,5}=3.6$, $\left.\mathrm{H}_{5}\right), 3.67\left(3 \mathrm{H}, \mathrm{s}, \mathrm{CH}_{3} \mathrm{O}\right), 4.03\left(1 \mathrm{H}, \mathrm{dd}, J_{10,10}=8.7 \mathrm{~Hz}, J_{10,9}=4.6 \mathrm{~Hz}, \mathrm{H}_{10}\right), 4.08\left(1 \mathrm{H}, \mathrm{dd}, J_{10,10}=8.7 \mathrm{~Hz}\right.$, $\left.J_{10,9}=6.2 \mathrm{~Hz}, \mathrm{H}_{10}\right), 4.29\left(1 \mathrm{H}, \mathrm{m}, \mathrm{H}_{3}\right), 4.38\left(1 \mathrm{H}, \mathrm{ddd}, J_{9,8}=7.5 \mathrm{~Hz}, J_{10,9}=6.2 \mathrm{~Hz}, J_{10,9}=4.6 \mathrm{~Hz}, \mathrm{H}_{9}\right), 4.67$ $\left(1 \mathrm{H}, \mathrm{dd}, J_{7,6}=6.1 \mathrm{~Hz}, J_{6,5}=3.6 \mathrm{~Hz}, \mathrm{H}_{6}\right), 4.73\left(1 \mathrm{H}, \mathrm{dd}, J_{7,6}=6.1 \mathrm{~Hz}, J_{8,7}=3.6 \mathrm{~Hz}, \mathrm{H}_{7}\right), 4.93(1 \mathrm{H}, \mathrm{m}, \mathrm{NH})$, $5.12\left(1 \mathrm{H}, \mathrm{dd}, J_{2,1 \text { cis }}=10.4 \mathrm{~Hz}, J_{3,1 \text { cis }}=1.3 \mathrm{~Hz}, \mathrm{H}_{1 \text { cis }}\right), 5.21\left(1 \mathrm{H}, \mathrm{dd}, J_{2,1 \text { trans }}=17.0 \mathrm{~Hz}, J_{3,1 \text { trans }}=1.2 \mathrm{~Hz}\right.$, $\left.\mathrm{H}_{1 \text { trans }}\right), 5.80\left(1 \mathrm{H}\right.$, ddd, $\left.J_{2,1 \text { trans }}=17.0 \mathrm{~Hz}, J_{2,1 \text { cis }}=10.4 \mathrm{~Hz}, J_{3,2}=5.6 \mathrm{~Hz}, \mathrm{H}_{2}\right) ;{ }^{13} \mathrm{C}-\mathrm{NMR}: \delta 24.6,25.2,25.8$, 26.9, 33.7, 51.4, 52.1, 66.9, 73.1, 79.5, 80.6, 81.4, 81.7, 109.1, 112.4, 114.8, 138.5, 156.6; Anal. Calcd for $\mathrm{C}_{18} \mathrm{H}_{29} \mathrm{NO}_{7}$ (371.43): C 58.21, H 7.87, N 3.77; found C 58.05, H 7.54, N 3.53.

\section{4,7-Anhydro-2,3-dideoxy-5,6:8,9-di-O-isopropylidene-2(S)-(methoxycarbonylamino)-D-glycero-D-}

galacto-nononic acid (12a): A solution of $\mathbf{1 0 a}(0.28 \mathrm{~g}, 0.76 \mathrm{mmol})$ in methanol $(28 \mathrm{~mL})$ was cooled to $-78{ }^{\circ} \mathrm{C}$. Ozone was then passed through the solution under vigorous stirring. The maximum time for the ozone treatment was $30 \mathrm{~min}$. This resulted in the formation of a bluish solution. Dry nitrogen was passed through the cold solution in order to remove excess ozone. $\mathrm{Ph}_{3} \mathrm{P}(0.20 \mathrm{~g}, 0.76 \mathrm{mmol})$ and $\mathrm{CH}_{2} \mathrm{Cl}_{2}(11 \mathrm{~mL})$ were added and the solution was allowed to warm up to room temperature while stirring was continued for $1.5 \mathrm{~h}$. The solvent was removed under reduced pressure and the chromatography of the residue (hexane-ethyl acetate, $2: 1$ ) afforded $0.25 \mathrm{~g}(87 \%)$ of $11 \mathrm{a}$ as a colorless oil which was used immediately in the next step. ${ }^{1} \mathrm{H}-\mathrm{NMR}: \delta 1.32\left(3 \mathrm{H}, \mathrm{s}, \mathrm{CH}_{3}\right), 1.38\left(3 \mathrm{H}, \mathrm{s}, \mathrm{CH}_{3}\right)$, $1.44\left(3 \mathrm{H}, \mathrm{s}, \mathrm{CH}_{3}\right), 1.47\left(3 \mathrm{H}, \mathrm{s}, \mathrm{CH}_{3}\right), 2.07-2.17\left(1 \mathrm{H}, \mathrm{m}, \mathrm{H}_{3}\right), 2.24\left(1 \mathrm{H}, \mathrm{m}, \mathrm{H}_{3}\right), 3.54\left(1 \mathrm{H}, \mathrm{dd}, J_{8,7}=6.8\right.$ $\left.\mathrm{Hz}, J_{7,6}=3.7 \mathrm{~Hz}, \mathrm{H}_{7}\right), 3.62-3.68\left(1 \mathrm{H}, \mathrm{m}, \mathrm{H}_{4}\right), 3.70\left(3 \mathrm{H}, \mathrm{s}, \mathrm{CH}_{3} \mathrm{O}\right), 4.03\left(1 \mathrm{H}, \mathrm{dd}, J_{9,9}=8.7 \mathrm{~Hz}, J_{9,8}=4.8\right.$ $\left.\mathrm{Hz}, \mathrm{H}_{9}\right), 4.08\left(1 \mathrm{H}, \mathrm{dd}, J_{9,9}=8.7 \mathrm{~Hz}, J_{9,8}=6.3 \mathrm{~Hz}, \mathrm{H}_{9}\right), 4.32-4.40\left(2 \mathrm{H}, \mathrm{m}, \mathrm{H}_{2}, \mathrm{H}_{8}\right), 4.60\left(1 \mathrm{H}, \mathrm{dd}, J_{6,5}=6.1\right.$ $\left.\mathrm{Hz}, J_{5,4}=3.7 \mathrm{~Hz}, \mathrm{H}_{5}\right), 4.74\left(1 \mathrm{H}, \mathrm{dd}, J_{6,5}=6.1 \mathrm{~Hz}, J_{7,6}=3.7 \mathrm{~Hz}, \mathrm{H}_{6}\right), 5.77(1 \mathrm{H}, \mathrm{m}, \mathrm{NH}), 9.64(1 \mathrm{H}, \mathrm{bs}$, $\mathrm{CH}=\mathrm{O})$. A solution of $\mathrm{NaClO}_{2}(80 \%, 0.57 \mathrm{~g}, 6.3 \mathrm{mmol})$ and $\mathrm{NaH}_{2} \mathrm{PO}_{4}(0.71 \mathrm{~g}, 4.5 \mathrm{mmol})$ in $3.8 \mathrm{~mL}$ of water was added dropwise to a solution of aldehyde 11a $(0.25 \mathrm{~g}, 0.68 \mathrm{mmol})$ in acetonitrile/tert-butyl 
alcohol/2-methyl-2-butene $(15 \mathrm{~mL}, 4: 4: 1)$ at $0{ }^{\circ} \mathrm{C}$ over $5 \mathrm{~min}$ and stirred at the same temperature for 35 min. The reaction mixture was poured into brine $(12 \mathrm{~mL})$ and extracted with ethyl acetate $(2 \times 25 \mathrm{~mL})$. The combined organic layers were dried $\left(\mathrm{Na}_{2} \mathrm{SO}_{4}\right)$. The solvent was removed under reduced pressure and chromatography of the residue on silica gel (hexane-ethyl acetate, 1:2) afforded $0.20 \mathrm{~g} \mathrm{(74 \% )} \mathrm{of}$ carboxylic acid 12a as a colorless oil; $[\alpha]_{\mathrm{D}}{ }^{25}=-33\left(c 0.12, \mathrm{CHCl}_{3}\right) ;{ }^{1} \mathrm{H}-\mathrm{NMR}: \delta 1.31\left(3 \mathrm{H}, \mathrm{s}, \mathrm{CH}_{3}\right), 1.37$ $\left(3 \mathrm{H}, \mathrm{s}, \mathrm{CH}_{3}\right), 1.44\left(3 \mathrm{H}, \mathrm{s}, \mathrm{CH}_{3}\right), 1.45\left(1 \mathrm{H}, \mathrm{s}, \mathrm{CH}_{3}\right), 2.18-2.26\left(2 \mathrm{H}, \mathrm{m}, \mathrm{H}_{3}\right), 3.51\left(1 \mathrm{H}, \mathrm{dd}, J_{8,7}=6.8 \mathrm{~Hz}\right.$, $\left.J_{7,6}=3.6 \mathrm{~Hz}, \mathrm{H}_{7}\right), 3.62-3.66\left(4 \mathrm{H}, \mathrm{m}, \mathrm{H}_{4}, \mathrm{CH}_{3} \mathrm{O}\right), 4.02-4.08\left(2 \mathrm{H}, \mathrm{m}, \mathrm{H}_{9}\right), 4.37\left(1 \mathrm{H}, \mathrm{ddd}, J_{8,7}=6.8 \mathrm{~Hz}\right.$, $\left.J_{9,8}=6.1 \mathrm{~Hz}, J_{9,8}=4.8 \mathrm{~Hz}, \mathrm{H}_{8}\right), 4.48\left(1 \mathrm{H}, \mathrm{m}, \mathrm{H}_{2}\right), 4.59\left(1 \mathrm{H}, \mathrm{dd}, J_{6,5}=6.1 \mathrm{~Hz}, J_{5,4}=3.7 \mathrm{~Hz}, \mathrm{H}_{5}\right), 4.70(1 \mathrm{H}$, $\left.\mathrm{dd}, J_{6,5}=6.1 \mathrm{~Hz}, J_{7,6}=3.6 \mathrm{~Hz}, \mathrm{H}_{6}\right), 5.76\left(1 \mathrm{H}, \mathrm{d}, J_{2, \mathrm{NH}}=7.9 \mathrm{~Hz}, \mathrm{NH}\right) ;{ }^{13} \mathrm{C}-\mathrm{NMR}: \delta 24.6,25.3,25.7,26.9$, $30.9,52.1,52.3,66.7,73.1,80.0,2 \times 81.0,81.6,109.2,112.5,156.7,171.2$; Anal. Calcd for $\mathrm{C}_{17} \mathrm{H}_{27} \mathrm{NO}_{9}$ (389.40): C 52.44, H 6.99, N 3.60; found C 52.10, H 7.00, N 3.41.

\section{4,7-Anhydro-2,3-dideoxy-5, 6:8,9-di-O-isopropylidene-2(R)-(methoxycarbonylamino)-D-glycero-D-}

galacto-nononic acid (12b): A solution of $\mathbf{1 0 b}(185 \mathrm{mg}, 0.498 \mathrm{mmol})$ in methanol (18 $\mathrm{mL})$ was cooled to $-78{ }^{\circ} \mathrm{C}$. Ozone was then passed through the solution under vigorous stirring. The maximum time for the ozone treatment was $30 \mathrm{~min}$. This resulted in the formation of a bluish solution. Dry nitrogen was passed through the cold solution in order to remove excess ozone. $\mathrm{Ph}_{3} \mathrm{P}(0.13 \mathrm{~g}, 0.498 \mathrm{mmol})$ and $\mathrm{CH}_{2} \mathrm{Cl}_{2}(7 \mathrm{~mL})$ were added and the solution was allowed to warm up to room temperature while stirring was continued for $1.5 \mathrm{~h}$. The solvent was removed under reduced pressure and the chromatography of the residue (hexane-ethyl acetate, $2: 1$ ) gave $0.16 \mathrm{~g}(88 \%)$ of aldehyde $\mathbf{1 1 b}$ as a colorless oil which was used immediately in the next step. ${ }^{1} \mathrm{H}-\mathrm{NMR}: \delta 1.33\left(3 \mathrm{H}, \mathrm{s}, \mathrm{CH}_{3}\right), 1.36(3 \mathrm{H}, \mathrm{s}$, $\left.\mathrm{CH}_{3}\right), 1.43\left(3 \mathrm{H}, \mathrm{s}, \mathrm{CH}_{3}\right), 1.48\left(3 \mathrm{H}, \mathrm{s}, \mathrm{CH}_{3}\right), 2.11-2.21\left(1 \mathrm{H}, \mathrm{m}, \mathrm{H}_{3}\right), 2.26-2.36\left(1 \mathrm{H}, \mathrm{m}, \mathrm{H}_{3}\right), 3.48(1 \mathrm{H}$, $\left.\mathrm{dd}, J_{8,7}=7.0 \mathrm{~Hz}, J_{7,6}=3.6 \mathrm{~Hz}, \mathrm{H}_{7}\right), 3.62-3.67\left(1 \mathrm{H}, \mathrm{m}, \mathrm{H}_{4}\right), 3.70\left(3 \mathrm{H}, \mathrm{s}, \mathrm{CH}_{3} \mathrm{O}\right), 3.94\left(1 \mathrm{H}, \mathrm{dd}, J_{9,9}=8.7 \mathrm{~Hz}\right.$, $\left.J_{9,8}=4.8 \mathrm{~Hz}, \mathrm{H}_{9}\right), 4.04\left(1 \mathrm{H}, \mathrm{dd}, J_{9,9}=8.7 \mathrm{~Hz}, J_{9,8}=6.2 \mathrm{~Hz}, \mathrm{H}_{9}\right), 4.32-4.36\left(2 \mathrm{H}, \mathrm{m}, \mathrm{H}_{2}, \mathrm{H}_{8}\right), 4.68(1 \mathrm{H}, \mathrm{dd}$, $\left.J_{6,5}=6.1 \mathrm{~Hz}, J_{5,4}=3.5 \mathrm{~Hz}, \mathrm{H}_{5}\right), 4.74\left(1 \mathrm{H}, \mathrm{dd}, J_{6,5}=6.1 \mathrm{~Hz}, J_{7,6}=3.6 \mathrm{~Hz}, \mathrm{H}_{6}\right), 5.63(1 \mathrm{H}, \mathrm{m}, \mathrm{NH}), 9.52(1 \mathrm{H}$, bs, $\mathrm{CH}=\mathrm{O})$. A solution of $\mathrm{NaClO}_{2}(80 \%, 0.37 \mathrm{~g}, 4.1 \mathrm{mmol})$ and $\mathrm{NaH}_{2} \mathrm{PO}_{4}(0.46 \mathrm{~g}, 2.9 \mathrm{mmol})$ in $2.5 \mathrm{~mL}$ of water was added dropwise to a solution of aldehyde $11 \mathrm{~b}(0.16 \mathrm{~g}, 0.43 \mathrm{mmol})$ in acetonitrile/tertbutyl alcohol/2-methyl-2-butene $(10 \mathrm{~mL}, 4: 4: 1)$ at $0{ }^{\circ} \mathrm{C}$ over $5 \mathrm{~min}$ and stirred at the same temperature for $45 \mathrm{~min}$. The reaction mixture was poured into brine $(8 \mathrm{~mL})$ and extracted with ethyl acetate $(2 \times 16$ $\mathrm{mL})$. The combined organic layers were dried $\left(\mathrm{Na}_{2} \mathrm{SO}_{4}\right)$. The solvent was removed under reduced pressure and the chromatography of the residue on silica gel (hexane-ethyl acetate, $1: 2$ ) gave $0.12 \mathrm{~g}$ $(73 \%)$ of carboxylic acid $\mathbf{1 2 b}$ as a white viscous oil; $[\alpha]_{\mathrm{D}}{ }^{25}=-7\left(c 0.49, \mathrm{CHCl}_{3}\right) ;{ }^{1} \mathrm{H}-\mathrm{NMR}: \delta 1.32(3 \mathrm{H}$, $\left.\mathrm{s}, \mathrm{CH}_{3}\right), 1.35\left(3 \mathrm{H}, \mathrm{s}, \mathrm{CH}_{3}\right), 1.42\left(3 \mathrm{H}, \mathrm{s}, \mathrm{CH}_{3}\right), 1.45\left(3 \mathrm{H}, \mathrm{s}, \mathrm{CH}_{3}\right), 2.05-2.13\left(1 \mathrm{H}, \mathrm{m}, \mathrm{H}_{3}\right), 2.29-2.36(1 \mathrm{H}$, $\left.\mathrm{m}, \mathrm{H}_{3}\right), 3.44\left(1 \mathrm{H}, \mathrm{m}, \mathrm{H}_{7}\right), 3.60-3.63\left(1 \mathrm{H}, \mathrm{m}, \mathrm{H}_{4}\right), 3.65\left(3 \mathrm{H}, \mathrm{s}, \mathrm{CH}_{3} \mathrm{O}\right), 3.98-4.04\left(2 \mathrm{H}, \mathrm{m}, \mathrm{H}_{9}\right), 4.34(1 \mathrm{H}$, ddd, $\left.J_{8,7}=7.3 \mathrm{~Hz}, J_{9,8}=6.2 \mathrm{~Hz}, J_{9,8}=5.4 \mathrm{~Hz}, \mathrm{H}_{8}\right), 4.39-4.40\left(1 \mathrm{H}, \mathrm{m}, \mathrm{H}_{2}\right), 4.70\left(2 \mathrm{H}, \mathrm{m}, \mathrm{H}_{5}, \mathrm{H}_{6}\right), 5.61(1 \mathrm{H}$, d, $\left.J_{2, \mathrm{NH}}=7.1 \mathrm{~Hz}, \mathrm{NH}\right) ;{ }^{13} \mathrm{C}-\mathrm{NMR}: \delta 24.7,25.3,25.8,26.9,31.4,51.9,52.1,66.8,73.1,80.7,2$ x 81.4, 81.6, 109.0, 112.4, 156.7, 173.4; Anal. Calcd for $\mathrm{C}_{17} \mathrm{H}_{27} \mathrm{NO}_{9}$ (389.40): C 52.44, H 6.99, N 3.60; found C 52.68, H 7.03, N 3.82 . 


\section{Crystal structure determination of $\mathbf{8 a}$}

A single crystal of 8a suitable for X-ray structure analysis was prepared by growth under slow evaporation of a mixture of diethyl ether and hexane at room temperature in a form of the colorless prisms. The intensities were collected at $295 \mathrm{~K}$ on a diffractometer Oxford Diffraction Gemini R CCD using Mo-K $\alpha$ radiation (0.71073 $\AA$ ). Details of crystal data, data collection and refinement parameters are given in Table 1.

Table 1. Crystal and experimental data for compound 8a

\begin{tabular}{|c|c|}
\hline Empirical formula & $\mathrm{C}_{17} \mathrm{H}_{25} \mathrm{~N}_{1} \mathrm{O}_{5} \mathrm{~S}_{1}$ \\
\hline Formula weight & 355.46 \\
\hline Temperature, $T(\mathrm{~K})$ & $100 \mathrm{~K}$ \\
\hline Wavelength, $\lambda(\AA)$ & 0.71093 \\
\hline Crystal system & Trigonal \\
\hline Space group & P31 21 \\
\hline \multirow[t]{3}{*}{ Unit cell dimensions $(\AA)$} & $a=10.4026(2) \gamma=120^{\circ}$ \\
\hline & $b=10.4026(2)$ \\
\hline & $c=30.8227(5)$ \\
\hline Unit-cell volume, $V\left(\AA^{3}\right)$ & $2889(1)$ \\
\hline Formula units per unit cell, $Z$ & 6 \\
\hline Calculated density, $D_{x}\left(\mathrm{~g} \mathrm{~cm}^{-3}\right)$ & 1.233 \\
\hline Absorption coefficient, $\mu\left(\mathrm{mm}^{-1}\right)$ & 0.192 \\
\hline $\mathrm{F}(000)$ & 744 \\
\hline Crystal size (mm) & $0.630 \times 0.085 \times 0.050$ \\
\hline Theta range for data collection, $\left({ }^{\circ}\right)$ & $3.00-29.47$ \\
\hline Index ranges & $-12 \leq \mathrm{h} \leq 12,-12 \leq \mathrm{k} \leq 12,-38 \leq 1 \leq 38$ \\
\hline Independent reflections $[I>2 \sigma(I)]$ & $3483(\operatorname{Rint}=0.048)$ \\
\hline Absorption correction & Empiric Psi-scan \\
\hline Max. and min. transmission & 0.927 and 0.966 \\
\hline Refinement method & Full-matrix least-squares on $\mathrm{F}^{2}$ \\
\hline Data / parameters & $3922 / 263$ \\
\hline Goodness-of-fit (all) & 1.09 \\
\hline Final $\mathrm{R}$ indices $[I>2 \sigma(I)]$ & $R 1=0.0480, \mathrm{w} R 2=0.0119$ \\
\hline $\mathrm{R}$ indices (all data) & $R 1=0.0527, \mathrm{w} R 2=0.1212$ \\
\hline Largest diff. peak and hole & 0.33 and $-0.37\left(\mathrm{e} \AA^{-3}\right)$ \\
\hline
\end{tabular}

The structure was solved by direct methods [16]. All non-hydrogen atoms were refined anisotropically by full-matrix least-squares calculations based on F2 [16]. The hydrogen atoms bonded to nitrogen atoms were found in a difference Fourier map and their coordinates and isotropic thermal parameters have been refined freely. All other hydrogen atoms were included in calculated positions as 
riding atoms, with SHELXL97 [16] defaults. PLATON [17] program was used for structure analysis and molecular and crystal structure drawings preparation. The following crystal structure has been deposited at the Cambridge Crystallographic Data Centre and allocated the deposition number CCDC 697340. These data can be obtained free of charge via www.ccdc.cam.ac.uk/data_request/cif, by emailing data_request@ccdc.cam.ac.uk, or by contacting The Cambridge Crystallographic Data Centre, 12, Union Road, Cambridge CB2 1EZ, UK; fax: +44 1223336033.

\section{Acknowledgements}

The present work was supported by the Grant Agency (No. 1/0281/08, No. 1/3557/06, No. 1/0817/08) of the Ministry of Education, the Research and Development Support Agency (APVV No. 20-038405), Slovak Republic and COST Action D32/011/05 Chemistry in High-Energy Microenvironments.

\section{References}

1. Dwek, R. A. Glycobiology: Toward Understanding the Function of Sugars. Chem. Rev. 1996, 96, 683-720.

2. Dondoni, A.; Marra, A. Methods for Anomeric Carbon-Linked and Fused Sugar Amino Acid Synthesis: To Gateway to Artificial Glycopeptides. Chem. Rev. 2000, 100, 4395-4421 and references cited therein.

3. Gruner, S. A. W.; Locardi, E.; Lohof, E.; Kessler, H. Carbohydrate-Based Mimetics in Drug Design: Sugar Amino Acids and Carbohydrate Scaffolds. Chem. Rev. 2002, 102, 491-514 and references cited therein.

4. Schweizer, F. Glycosamino Acids: Building Blocks for Combinatorial Synthesis-Implications for Drug Discovery. Angew. Chem. Int. Ed. 2002, 41, 230-253.

5. Risseeuw, M. D. P.; Overhand, M.; Fleet, G. W. J.; Simone, M. I. A compendium of sugar amino acids (SAA): scaffolds, peptide- and glyco-mimetics. Tetrahedron Asymmetry 2007, 18, 20012010 and references cited therein.

6. McGarvey, G. J.; Benedum, T. E.; Schmidtmann, F. W. Development of Co- and PostTranslational Synthetic Strategies to $C$-Neoglycopeptides. Org. Lett. 2002, 4, 3591-3594.

7. Colombo, L.; Giacomo, M.; Ciceri, P. Practical stereoselective synthesis of $\alpha$-D-C-mannosyl- $(R)$ alanine. Tetrahedron 2002, 58, 9381-9386.

8. Chambers, D. J.; Evans, G. R.; Fairbanks, A. J. Synthesis of $C$-gycosyl amino acids: scope and limitations of tandemTebbe/Claisen approach. Tetrahedron Asymmetry 2005, 16, 45-55.

9. Schmidtmann, F. W.; Benedum, T. E.; McGarvey, G. J. The preparation of $C$-glycosyl amino acids - an examination of olefin cross-metathesis. Tetrahedron Lett. 2005, 46, 4677-4681.

10. Yayakanthan, K.; Vankar, Y. D. Hybrid sugars as glycosidase inhibitors en route to 2-deoxy-2amino $C$-glycosyl amino acids. Tetrahedron Lett. 2006, 47, 8667-8671.

11. Shing, T. K. M.; Tsui, H.-C. Enantiospecific Syntheses of $(3 S, 4 R)$ - and $(3 S, 4 R, 7 S)$ Diastereoisomers of Goniofufurone. Tetrahedron Asymmetry 1994, 5, 1269-1274. 
12. Martinková, M.; Gonda, J.; Raschmanová, J. Novel Furanoid $\alpha$-Substituted $\alpha$-Amino Acid as a Potent Turn Mimic in Peptide Synthesis. Molecules 2006, 11, 564.

13. Gonda, J.; Martinková, M.; Raschmanová, J.; Balentová, E. Creation of quarternary stereocentres via [3,3]-sigmatropic rearrangement of allylic thiocyanates. A synthetic approach to $(+)$-myriocin. Tetrahedron Asymmetry 2006, 17, 1875-1882.

14. More, J. D.; Finney, N. A simple and Advantageous Protocol for the Oxidation of Alcohols with $o$-Iodoxybenzoic Acid. Org. Lett. 2002, 4, 3001-3003.

15. Kniežo, L.; Bernát, J.; Martinková, M. Synthesis of 2-Isothiocyanatotetrahydropyran and Its Reactions with Amines and Alcohols. Chem. Pap. 1994, 48, 103-107.

16. Sheldrick, G. M. A short history of SHELX. Acta Crystallogr. 2008, A64, 112-122.

17. Spek, A. L. Single-crystal structure validation with the program PLATON. J. Appl. Crystallogr. 2003, 36, 7-13.

Sample Availability: Samples of the compounds $\mathbf{7 , 8 a}$ and $\mathbf{8 b}$ are available from the authors.

(C) 2008 by the authors; licensee Molecular Diversity Preservation International, Basel, Switzerland. This article is an open-access article distributed under the terms and conditions of the Creative Commons Attribution license (http://creativecommons.org/licenses/by/3.0/). 